\title{
Médiévales
}

Langues, Textes, Histoire

69 | automne 2015

Travailler à Paris (XIIIe-XVIe siècle)

\section{Les registres de la chancellerie angevine de Naples. Un exemple de destruction et reconstitution de sources archivistiques à travers les siècles}

The Registers of the Angevin Archive of Naples : an Example of Destruction and Reconstruction of Archive's Source through the Centuries

\section{Gian Luca Borghese}

\section{OpenEdition \\ Journals}

Édition électronique

URL : https://journals.openedition.org/medievales/7640

DOI : 10.4000/medievales.7640

ISSN : $1777-5892$

\section{Éditeur}

Presses universitaires de Vincennes

\section{Édition imprimée}

Date de publication : 30 novembre 2015

Pagination : 171-182

ISBN : 978-2-84292-444-7

ISSN : 0751-2708

\section{Référence électronique}

Gian Luca Borghese, «Les registres de la chancellerie angevine de Naples. Un exemple de destruction et reconstitution de sources archivistiques à travers les siècles ", Médiévales [En ligne], 69 | automne 2015, mis en ligne le 30 novembre 2017, consulté le 24 avril 2022. URL : http://

journals.openedition.org/medievales/7640; DOI : https://doi.org/10.4000/medievales.7640 
Gian Luca Borghese

\section{Les registres de la chancellerie angevine de Naples \\ Un exemple de destruction et reconstitution de sources archivistiques à travers les siècles}

Une réflexion sur le parcours mouvementé des registres, et des archives en général, de la chancellerie angevine de Naples à travers les siècles ${ }^{1}$ peut être utile pour envisager non seulement la méthode d'analyse et d'interprétation d'une pareille source médiévale, mais aussi la variété des difficultés que peuvent affronter les sources archivistiques dans le cours de leur existence et les remèdes auxquels peuvent recourir les gardiens de ces mêmes sources afin que les archives médiévales continuent à être le miroir de celui qui les a produites et de son temps, dans le cadre du rapport dialectique particulier qui régit la relation entre les historiens et les sources archivistiques. Rapport, comme on cherchera à le mettre en évidence, instable, dépendant des conditions de conservation des sources elles-mêmes comme de la perception et de l'évaluation qu'en font les historiens. En effet, les archives ne peuvent pas seulement disparaitre par suite de leur destruction matérielle. Elles peuvent également cesser d'avoir une valeur juridique et scientifique quand les conditions dans lesquelles elles sont venues à se trouver les rendent précaires et compromettent pour ainsi dire leur réputation. Dans la tradition anglo-saxonne, par exemple, un document qui a été soustrait à la série archivistique à laquelle il appartenait, même s'il a pu être par un heureux hasard racheté sur le marché des antiquités, n'a plus de valeur juridique probante (il s'agit du principe de la unbroken custody), tandis que les interpolations systématiques de documents douteux risquent de décrédibiliser scientifiquement d'antiques fonds d'archives encore très prestigieux.

1. Sur les chancelleries de l'Italie méridionale au bas Moyen Âge, $c f$. en général R. DelLe DonNe, « Le cancellerie dell'Italia meridionale, sec. XIII-XV », Ricerche Storiche, 24 (1994), p. 361-388. 
Les archives de la chancellerie angevine de Naples furent transportées par mesure de protection, durant la seconde guerre mondiale, des Archives d'État de Naples à la villa Montesano de San Paolo Belsito de Nola, non loin de Naples, où elles furent entièrement détruites, à l'exception de quelques fragments, par suite de l'incendie des locaux où elles avaient été abritées, incendie opéré par ordre du commandement allemand le 30 septembre 1943. Cette catastrophe finale fut l'épilogue d'une histoire archivistique plutôt turbulente, parsemée de rebondissements. La concentration de la série archivistique la plus importante de la chancellerie angevine (celle des registres) à Bari, dans les Pouilles, sur ordre de Charles Ir d'Anjou, remontait à 1284 , et avait été accompagnée d'un ordre d'en exécuter l'inventaire ${ }^{2}$. On se trouvait alors tout près des origines du pouvoir dont elle émanait, puisque Charles I ${ }^{\text {er }}$, frère de saint Louis, était le fondateur de cette dynastie dont l'installation manu militari sur le trône de Sicile en 1266 avait été appuyée par la papauté dans sa lutte contre les souverains Hohenstaufen et dont l'empreinte originale, associant une composante française à la récupération de traits caractéristiques du royaume sicilien fondé par les Normands, allait marquer pendant deux siècles l'histoire méditerranéenne en général et, en particulier, celle des rapports entre la France, la Provence et l'Italie.

Sous le règne de Charles II d'Anjou, dès 1290, les registres furent progressivement regroupés à Naples. Vers 1292-1293, ils furent transférés, ainsi que le reste des archives de la chancellerie angevine et les bureaux de la Chambre des comptes, dans un palais appartenant à la famille Fieschi à coté de la Monnaie royale, léguant ainsi aux archives angevines le nom d'Archives de la Monnaie royale («Archivio della Regia Zecca »). En 1332, le roi Robert d'Anjou, petit-fils de Charles I ${ }^{\text {er }}$, acheta un palais près de l'église des Augustins, depuis appelée Sant'Agostino della Zecca, et y fit transférer les archives ${ }^{3}$. Dans ce nouvel emplacement, dès février 1336, quatre ans par conséquent après ce transfert, les eaux d'une pluie torrentielle inondèrent les locaux où étaient gardées ces « écritures », provoquant ainsi les premiers grands dégâts sur les fonds dont on ait conservé la trace ${ }^{4}$. Peu après, en 1346, une révolte populaire causée par l'assassinat d'André de Hongrie, mari de la reine Jeanne $\mathrm{I}^{\mathrm{re}}$ d'Anjou, se solda par la destruction

2. J. MAZZOLENI, Storia della ricostruzione della Cancelleria angioina 1265-1434, Naples, 1987 (Testi e documenti di storia napoletana, 37), p. 1-2 ; S. PALMIERI, « L'Archivio della Regia Zecca », dans ID., Degli Archivi napolitani. Storia e tradizione, Naples, 2002, p. 324 ; ID., « I registri della cancelleria angioina », dans G. Musca éd., Le eredità normannosveve nell'età angioina : persistenze e mutamenti nel Mezzogiorno. Atti delle quindicesime giornate normanno-sveve (Bari, 22-25 ottobre 2002), Bari, 2004, p. 383.

3. J. MAZzoleni, Storia della ricostruzione..., p. 2 ; S. PALMIERI, « I registri... », p. 383-384.

4. C. MINIERI RICCIO, Brevi notizie intorno all'Archivio angioino di Napoli, dopo le quali si pubblica per la prima volta parte di quei registri ora non più esistenti, Naples, 1862, p. IX. 
d'une autre section des archives angevines, celle de la comptabilité générale du royaume ${ }^{5}$, tandis qu'en 1348 l'occupation par Louis de Hongrie du Castel Nuovo, où était conservée une partie des registres des Maîtres rationnaires («Maestri rationali »), provoqua leur perte.

Entre 1540 et 1542, le vice-roi don Pedro de Tolède fit transférer les archives dans le château de Capuana (Castel Capuano), restauré et destiné par lui à abriter également les tribunaux. Les registres angevins reçurent une reliure (d'après Giambattista Bolvito, en 1556), qui les classait par nom du souverain et date des actes. Un nouvel inventaire général, en 1568, décrivit l'organisation du fonds d'archives en 436 volumes, devenus 444 volumes par suite de quelques nouvelles acquisitions en $1585^{6}$.

Il ne s'agissait plus toutefois, à ce point, des unités archivistiques d'origine, c'est-à-dire des registres tels qu'ils avaient été originellement produits par la chancellerie angevine : leur aspect avait été profondément altéré. Les pages qui s'étaient détachées au fil du temps avaient été reliées dans divers volumes sans tenir compte de leur contenu ; mais surtout, lors de la confection des nouveaux volumes au moment de la reliure, on était passé de l'organisation originale des registres sur la base de l'indiction, soit d'un cycle de quinze ans, à une organisation chronologique par année solaire et indiction, de sorte que de très nombreux registres finirent reliés dans le mauvais volume. L'homonymie de divers souverains angevins de Naples (Charles I ${ }^{\mathrm{er}}$, Charles II, Charles III, Jeanne $\mathrm{I}^{\mathrm{re}}$, Jeanne II) fut une circonstance aggravante, puisqu'elle favorisa la confusion des actes, émanant de l'un ou de l'autre, qui ne comportaient que la seule date indictionnelle ${ }^{7}$. Il s'agissait d'un dommage porté aux sources dû exclusivement à la méthode employée dans la reconstruction archivistique. À partir de la seconde moitié du $\mathrm{XVI}^{\mathrm{e}}$ siècle, les érudits responsables du fonds ne tardèrent pas à produire des répertoires pour faciliter la réinsertion in abstracto des documents dans leur contexte originel, compromis par les pertes et appariements sommaires. Mais il n'existait pas, à l'époque, de véritable distinction entre les « archivistes », qui avaient la garde des fonds, et les érudits, intéressés à l'étude des documents, puisqu'il s'agissait dans les deux cas du même groupe d'intellectuels.

La plus grave destruction matérielle avant la seconde guerre mondiale eut lieu en 1701, quand le peuple fut incité par suite de la conjuration dite « du prince de Macchia », du nom de son auteur, à prendre d'assaut le château de Capuana, où se trouvaient depuis l'époque du vice-roi don Pedro de Tolède les archives de la chancellerie, si bien que plusieurs volumes contenant les registres angevins furent jetés par les fenêtres et que l'on y mit

5. S. PALMIERI, « L'Archivio... », p. 326.

6. J. MAZZOLENI, Storia della ricostruzione ..., p. 3.

7. S. PALMIERI, « L'Archivio... », p. 328 ; ID., « I registri... », p. 384-385. 
le feu. Fut récupéré ce qui pouvait l'être, mais dans l'inventaire dressé par l'archiviste Michelangelo Chiarito dans la seconde moitié du XVIII ${ }^{\mathrm{e}}$ siècle, le nombre des volumes se trouvait réduit à trois cent cinquante ${ }^{8}$. À la fin du siècle fut planifiée une nouvelle opération de reliure des volumes, évidemment motivée par leur apparence, mais le travail de reliure fut l'occasion de démembrer et réapparier tout ce qui jusqu'alors était resté correctement relié. Les volumes ainsi «préparés » présentaient dorénavant de grands écarts chronologiques internes ${ }^{9}$; qui plus est, ils reçurent de nouveaux titres arbitraires, qui se substituèrent à ceux du XVI siècle à partir desquels les érudits étaient déjà habitués depuis deux siècles à citer les registres singuliers et leur contenu.

Finalement, en raison d'un décret royal de 1835, les archives angevines, ainsi que les autres archives napolitaines, furent concentrées dans le «Grande Archivio » de Naples, au sein de l'ancien monastère des Saints Severino et Sossio ${ }^{10}$. Le nouvel inventaire des Registri de la chancellerie angevine de 1854 fixait leur nombre à 378 (auxquels il faut ajouter 4 registres composés de fragments réliés par Bartolomeo Capasso et surnommés par conséquent « registri nuovi »), tous dotés d'un numéro de série arabe. Mais la table d'équivalence, créée sur la base des titres des volumes avec leurs reliures du XVIII ${ }^{\mathrm{e}}$ siècle, créait une confusion avec les inventaires $\mathrm{du} \mathrm{XVI}^{\mathrm{e}}$ et $\mathrm{du} \mathrm{XVII}^{\mathrm{e}}$ siècle et leurs titres. Le résultat fut que des registres encore existants étaient considérés comme perdus, alors qu'inversement

\section{J. MAZZOLENI, Storia della ricostruzione ..., p. 4.}

9. P. DURRIEU, Les Archives angevines de Naples. Étude sur les registres du roi Charles I Ir (1265-1285), Paris, t. I, 1886, p. 23 (Bibliothèque des Écoles françaises d'Athènes et de Rome, 46) : «Aucune règle fixe n'a présidé à la composition des volumes. Les débris des anciennes archives ont été reliés au hasard. Tantôt on a réuni deux ou trois registres primitifs conservés à peu près intacts. Tantôt on s'est borné à joindre ensemble des fragments informes ou des feuillets séparés. Nul souci de les classer ni de chercher à rapprocher les restes des cahiers primitifs. Tel des anciens registres est dispersé dans cinq ou six des volumes actuels ; tel autre se trouve inséré dans un seul volume ; mais il est mutilé, partagé en nombreux tronçons ; les feuillets sont intervertis, séparés à tout instant par de maladroites insertions, quelquefois même reliés à l'envers. »

10. J. MAZZOLEnI, Storia della ricostruzione..., p. 2 et n. 10. Au moment de leur entrée dans le « Grande Archivio », les Archives en question se composaient, à coté des « Registri » dont on a parlé jusqu'à présent, de deux autres séries, les Fascicoli et les Arche. Nos Registri étaient à l'origine, à leur tour, divisés en « Registri di Cancelleria et Registri di Camera ». Les premiers contenaient les actes politiques et administratifs de l'autorité royale, les deuxièmes les comptes des administrations locales soumis au contrôle des « Maestri Razionali ». Les « Fascicoli » étaient par contre des registres en papier (reliés au XIX ${ }^{\mathrm{e}}$ s. en 42 vol.) rédigés par les fonctionnaires auxquels s'adressaient les mandats royaux et contenant les actes administratifs et fiscaux liés à l'exécution des mêmes mandats. Les "Arche », finalement, en parchemin ou en papier, étaient à l'origine un recueil de documents originaux, publics ou privés, envoyés à l'administration centrale par les administrateurs locaux comme garantie de leurs comptes. Au XIX ${ }^{\mathrm{e}} \mathrm{s}$. ils furent reliés en volumes (49 pour les actes en parchemin, 20 pour ceux en papier). 
l'on considérait comme présents des registres qui avaient été entretemps perdus ${ }^{11}$. En somme, à la fin du XIX ${ }^{\mathrm{e}}$ siècle, les registres angevins étaient devenus une source extrêmement difficile à consulter et pratiquement inexploitable à cause de leur désordre et de leur caractère fragmentaire. Comme l'écrivait en 1896 l'historien italien Michelangelo Schipa : «Le chercheur le plus patient restait interdit devant le désordre et la confusion dans lesquels s'amassaient les documents des trois cent soixante dix-sept énormes volumes (souvent intitulés à partir d'un souverain et d'une année, tout en contenant des textes sans aucun rapport avec l'un ou l'autre, et exhibant régulièrement des documents tronqués, mutilés, et dont l'origine ne pouvait plus être retraçée). D'où la crainte permanente de ne pouvoir réussir à mener une enquête véritablement complète et exhaustive ${ }^{12}$.»

Ce fut d'abord un historien français, membre de l'École française de Rome alors nouvellement fondée, Paul Durrieu, qui tenta de remédier à cette situation dont il soulignait à son tour les nombreux désavantages pour le chercheur ${ }^{13}$. Dans son étude sur les registres de Charles $\mathrm{I}^{\text {er }}$, il rédigea un inventaire systématique de l'ensemble des unités qui reportaient effectivement des actes de Charles $I^{\text {er }}$ et tenta une reconstruction de ses registres originaires, subdivisés par l'année d'indiction ${ }^{14}$. Puis Bartolomeo Capasso, surintendant des Archives d'État de Naples à partir de 1882, procéda avec ses collaborateurs à la rédaction d'un inventaire analytique de chaque registre, intégrant tous les documents fragmentaires. Cette ouvre, publiée en 1894 sous le titre d'Inventario cronologico-sistematico dei Registri Angioini ${ }^{15}$ ( Inventaire chronologico-systématique des registres angevins »), rendit possible un nouvel épanouissement des études sur l'ère angevine entre la fin du XIX ${ }^{e}$ siècle et la première moitié du $\mathrm{XX}^{\mathrm{e}}$ siècle ${ }^{16}$. Les chercheurs réagirent en effet positivement aux résultats de cette reconstruction archivistique, de sorte que deux directions de recherche sur le Mezzogiorno angevin s'affirmèrent rapidement : une école française

11. S. PALMIERI, « L'Archivio... », p. 334 ; ID., « I registri...», p. 386-388.

12. M. SCHIPA, recension de l'Inventario cronologico-sistematico dei Registri Angioini, conservati nell'Archivio di Stato di Napoli de Bartolomeo Capasso, Archivio storico italiano, série V, 17 (1896), p. 175.

13. P. DURRIEU, Les Archives angevines...,t. I, avant-propos : «Malheureusement l'usage de ces archives est entravé par une confusion souvent extrême. Les difficultés deviennent surtout très grandes si l'on s'en prend aux plus anciens registres, à ceux qui remontent au règne de Charles $\mathrm{I}^{\mathrm{er}}$. Or, ces premiers registres, si pénibles à manier, sont, en même temps, les plus curieux à étudier sous le rapport de la diplomatique et de la paléographie, et, par leur contenu, les plus intéressants de beaucoup au point de vue français.»

14. L'œuvre, déjà citée n. 9, se compose de deux tomes : Les Archives angevines de Naples. Étude sur les registres du roi Charles I Ir (1265-1285), Paris, 1886-1887 (Bibliothèque des Écoles françaises d'Athènes et de Rome, 46 et 51).

15. B. CAPASSO, Inventario cronologico-sistematico dei Registri Angioini, conservati nell'Archivio di Stato di Napoli, Naples, 1894.

16. S. PALMIERI, «I registri... », p. 396-397. 
orientée essentiellement, mais non exclusivement, vers l'analyse des données de la vie socio-économique et administrative du royaume angevin (avec des historiens comme Paul Durrieu déjà nommé, Léon Cadier, Edouard Jordan, Georges Yver...), et une école italienne plus encline à l'enquête politico-institutionnelle (avec Giuseppe Del Giudice, Camillo Minieri Riccio, Francesco Cerone, Gennaro Maria Monti).

Quand survint la seconde guerre mondiale, la direction des Archives d'État de Naples était engagée dans des projets de reconstruction des registres angevins qui avaient été détruits dans le cours des catastrophes précédentes en s'aidant des plus anciens répertoires créés par les archivistes et érudits des $\mathrm{XVI}^{\mathrm{e}}-\mathrm{XVIII}{ }^{\mathrm{e}}$ siècles ; à peine le premier volume inspiré par le projet venait-il d'être publié, avec le titre Gli atti perduti della cancelleria angioina transuntati da Carlo De Lellis ${ }^{17}$ (que l'on pourrait traduire par «Transcriptions et résumés d'actes perdus de la chancellerie angevine par Carlo De Lellis »), que l'incendie de 1943 imposa, comme on peut l'imaginer, un radical changement de perspective.

Passé le premier moment de consternation, le surintendant des Archives d'État de Naples d'alors, Riccardo Filangieri, eut l'idée d'une solution en consultant les registres de la salle d'étude des Archives. Il apparaissait en effet que trois cent cinquante chercheurs avaient travaillé sur les registres angevins dans les quarante premières années du vingtième siècle. C'était un nombre énorme par rapport aux rythmes d'étude de ces années, avec une production de transcriptions et de copies équivalente ${ }^{18}$. Avec l'aide des archivistes des Archives d'État de Naples, réunis dans un Ufficio della Ricostruzione angioina («Bureau de la reconstruction angevine »), Riccardo Filangieri lança un ambitieux projet de reconstruction « idéale » des documents de l'antique chancellerie en utilisant les répertoires des anciens archivistes/archivari et les transcriptions, extraits et notes rédigés à partir des documents angevins par les chercheurs et érudits, les institutions ecclésiastiques, les communes ou les simples personnes privées à fins patrimoniales ou d'étude. Il allait donc falloir retrouver ces transcriptions ou extraits ( «sunti») dans les archives et bibliothèques publiques et privées, souvent auprès d'institutions ecclésiastiques ou dans les archives des érudits du XIX ${ }^{\mathrm{e}}$ siècle qui avaient fréquenté les Archives d'État de Naples. On pouvait y ajouter les actes originaux émis par la chancellerie angevine et subsistant encore dans les archives des destinataires, les divers recueils diplomatiques édités, et le patrimoine photographique et microfilmé qui

17. B. Mazzoleni éd., Atti perduti della Cancelleria angioina transuntati da Carlo De Lellis, 1939 (Regesta chartarum Italiae, XXV) et 1943 (Regesta chartarum Italiae, XXXI).

18. S. PALMIERI, « I registri... », p. 401. 
afflua à l'Ufficio della Ricostruzione grâce à la générosité des érudits contactés ${ }^{19}$.

R. Filangieri, pour la publication du travail de reconstruction des registres, se prononça en faveur d'une reconstruction idéale de l'ordre original des registres et des documents qu'ils contenaient afin de refléter leur production originale par la chancellerie : les actes suivent donc l'ordre qu'ils avaient hypothétiquement dans les registres originaires ${ }^{20}$. Le souvenir des aléas de la réorganisation des registres jusqu'à nos jours devait être en revanche garanti par la citation, au pied de chaque document, de la dernière cote qu'il avait reçue. On ne peut toutefois pas sous-évaluer les limites qui caractérisent une reconstruction du patrimoine documentaire ainsi organisée, car elle dépend des critères au moyen desquels les documents originaux ont été sélectionnés pour être transcrits, non en raison d'une aspiration impartiale à les conserver, mais pour s'en servir pour les raisons les plus variées ${ }^{21}$.

Cette entreprise débuta avec la parution en 1950 d'un premier volume contenant les six premiers registres reconstruits du règne de Charles ${ }^{\text {er }}$ d'Anjou ${ }^{22}$. Elle est arrivée en 2010 à la publication du volume cinquante, couvrant jusqu'à l'année 1295, la dixième du règne de Charles II d'Anjou ${ }^{23}$.

Quelle fut la réaction des historiens, d'abord face à la catastrophe de 1943, ensuite face à ce laborieux travail de reconstruction ? La destruction entrâna logiquement un rapide tarissement des recherches sur la dynastie angevine de Naples et la majeure partie des chercheurs préféra remonter en amont à l'époque du royaume normano-souabe, ou se projeter en aval, à l'époque de la substitution des Angevins par les Aragonais ${ }^{24}$. Quant à la maigre production historiographique sur les Angevins après la seconde

19. Ibid., p. 401-402.

20. Voir l'introduction de Riccardo Filangieri au volume I registri dalla Cancelleria angioina ricostruiti da $R$. Filangieri con la collaborazione degli archivisti napoletani, I (1265-1269), Naples, 1950, p. XI.

21. S. MoRelLI, Il controllo delle periferie nel Mezzogiorno angioino alla metà del XIII secolo : produzione e conservazione di carte, Reti Medievali 9 (2008), p. 28.

22. Voir n. 20.

23. Iregistri della Cancelleria angioina ricostruiti da R. Filangieri con la collaborazione degli archivisti napoletani, L (1267-1295), Naples, 1950-2010.

24. S. MoRELLI, « La storiografia sul Regno angioino di Napoli : una nuova stagione di studi », Studi storici 41/4 (2000), p. 1024. 
guerre mondiale, elle recourut à d'autres sources, qui n'étaient plus de nature administrative, mais artistique ${ }^{25}$ ou littéraire ${ }^{26}$.

Serena Morelli a attribué à la seconde moitié des années 1980 les premiers signes d'une inversion de tendance ${ }^{27}$. Celle-ci se manifeste notamment dans un essai de Giovanni Vitolo sur le royaume angevin de Naples destiné à l'ouvrage collectif Storia del Mezzogiorno $(1986)^{28}$, le catalogue d'une exposition documentaire tenue à Marseille $(1988)^{29}$, et l'organisation de deux importants colloques consacrés à L'État angevin en $1995^{30}$ et à La Noblesse dans les territoires angevins en $1998^{31}$. La reprise des études sur le Mezzogiorno angevin est assurément liée au fait qu'à la fin des années 1980 l'entreprise de reconstruction des registres mettait désormais à disposition des érudits un matériel non négligeable. La production historiographique de langue allemande, qui s'est récemment concentrée sur les mécanismes de l'administration et la fiscalité royale ${ }^{32}$, comme celle italo-française, intéressée qu'elle est par l'organisation judiciaire et la prosopographie du personnel administratif ${ }^{33}$, témoignent toutefois d'une

25. F. BologNA, Ipittori alla Corte angioina di Napoli, 1266-1414, e un riesame dell'arte nell'età fridericiana, Rome, 1969. Ce chercheur éminent, doté d'une capacité d'observation et d'une culture exceptionnelles, appartenait encore à cette génération d'historiens de l'art qui ne considérait pas le recours à l'enquête archivistique comme indispensable pour corroborer ses attributions.

26. A. BARBERO, « Il mito angioino nella cultura italiana e provenzale fra Duecento e Trecento », Bollettino storico-bibliografico subalpino, 79 (1981), p. 107-220, et 80 (1982), p. 389-450.

27. S. MoRELLI, Il "risveglio" della storiografia politico-istituzionale sul regno angioino di Napoli, en ligne : http://www.rmojs.unina.it/index.php/rm/article/view/217.

28. G. Vitolo, «Il Regno angioino », dans G. Galasso, R. Romeo éd., Storia del Mezzogiorno, IV-1, Rome, 1986, p. 9-86.

29. I. BonNot éd., Marseille et ses rois de Naples. La diagonale angevine. 1265-1382, Aix-en-Provence, 1988.

30. L'État angevin. Pouvoir, culture et société entre XIII et XIVe siècle. Actes du colloque international organisé par l'American Academy in Rome, l'École française de Rome, l'Istituto storico italiano per il Medio Evo, l'UMR Telemme et l'Université de Provence, l'Università degli Studi di Napoli «Federico II » (Rome/Naples, 7-11 novembre 1995), Rome, 1998 (Collection de l'École française de Rome, 245).

31. N. Coulet ET J.-M. MATZ éd., La Noblesse dans les territoires angevins à la fin du Moyen Âge. Actes du colloque international organisé par l'Université d'Angers (Angers/ Saumur, 3-6 juin 1998), Rome, 2000 (Collection de l'École française de Rome, 275).

32. A. KIESEWETTER, « La cancelleria angioina », dans L'État angevin..., p. 361-416.

33. S. MoRELLI, «Ad exstirpanda vitia : normativa regia e sistemi di controllo sul funzionariato nella prima età angioina », Mélanges de l'École française de Rome, Moyen Âge, 109/2 (1997), p. 463-475 ; D. CLÉMENT, « Juges et diplomates de la cour angevine de Naples », dans J.-P. BOYER, A. MALlOUX et L. VERDON éd., La Justice temporelle dans les territoires angevins, Actes du colloque de Aix-en-Provence (21-23 février 2002), Rome, 2005 (Collection de l'École française de Rome), p. 95-105 ; D. CARRAZ, « Pro servitio maiestatis nostre. Templiers et hospitaliers au service de la diplomatie de Charles Ir et Charles II », dans Z. KordÉ et I. PETROVICS éd., La Diplomatie des États Angevins aux XIII et XIVe siècles. Actes du colloque international de Szeged, Visegrád, Budapest (13-16 septembre 2007), 
tendance générale à ne guère aller au-delà de la période actuellement couverte par la reconstruction des registres de la chancellerie ${ }^{34}$. Dans cette optique, le volume de l'autrichien Andreas Kiesewetter, imposante et minutieuse étude consacrée à la seule première partie du règne de Charles II d'Anjou ${ }^{35}$, sans s'aventurer au-delà de l'année 1295, alors que la reconstruction des registres au moment de la publication de son travail avait à peine atteint les années 1292-1293, est au moins assez représentatif de la tendance historiographique qui assume sa dépendance à l'égard de la reconstruction archivistique et considère qu'elle est pratiquement incontournable. Dans le cas contraire, on privilégie l'étude de la cour, de la symbolique du pouvoir, de l'art et de la culture angevine, en recourant, de manière compréhensible, à des sources littéraires ou artistiques ${ }^{36}$. Il s'agit d'un choix bien représenté par le beau livre de l'américaine Samantha Kelly ${ }^{37}$. Celui-ci prend en effet pour objet la figure du roi Robert d'Anjou, en analysant à partir de sources littéraires et iconographiques les moyens employés dans la construction de son image royale.

L'objet et la problématique de mon étude sur les rapports entre le royaume angevin et la Méditerranée entre 1266 et $1282^{38}$ m'inscrivent plutôt

Rome/Szeged, 2010, p. 21-42 ; F. BÉRENGER, « Le rôle des compagnies toscanes dans la diplomatie de Charles II », ibid., p. 43-55 ; S. MoreLLI, « La territorializzazione della politica : competenze, metodi e obiettivi del personale amministrativo addetto alle inchieste », dans T. PÉCOUT éd., Quand gouverner c'est enquêter. Les pratiques politiques de l'enquête princière (Occident XIII ${ }^{e}$-XIV ${ }^{e}$ siècles). Actes du colloque international d'Aix-en-Provence et Marseille (19-21 mars 2009), Paris, 2010, p. 239-256.

34. D. CARRAZ, «Pro servitio maiestatis... », p. 23, n. 11, le déclare explicitement. Serena Morelli tente de minorer certains aspects de cette dépendance en rappelant la possibilité de recourir également à la consultation de séries documentaires originales conservées dans les archives de grandes familles, d'institutions ecclésiastiques et d'ordres religieux, sans sousévaluer l'importance, en particulier pour les recherches prosopographiques, des anciens répertoires comme celui de Chiarito, $c f$. S. MoRELLI, " La storiografia sul Regno... », p. 1041 ; EAD., Per conservare la pace. I Giustizieri del regno di Sicilia da Carlo I a Carlo II d'Angiò, Naples, 2012, p. XVI et 297.

35. A. KIESEWETTER, Die Anfänge der Regierung König Karls II. von Anjou (12781295). Das Königreich Neapel, die Grafschaft Provence und der Mittelmeerraum zu Ausgang des 13. Jahrhunderts, Husum, 1999 (Historische Studien, 451).

36. A. BARBERO, «La propaganda di Roberto d'Angiò re di Napoli (1309-1343) », dans P. CAmmarosano éd., Le forme della propaganda politica nel Due e nel Trecento, Rome, 1994 (Collection de l'École française de Rome, 201), p. 111-131 ; S. ASPERTI, Carlo I d'Angiò e i trovatori. Componenti provenzali e angioine nella tradizione manoscritta della lirica trobadorica, Ravenne, 1995 ; R. LIBRANDI, « Corte e cavalleria della Napoli angioina nel Teseida del Boccaccio », Medioevo romanzo, 4 (1997), p. 53-72 ; A. BARBERO, « Letteratura e politica fra Provenza e Napoli », dans L'État angevin..., p. 159-172 ; J.-P. BOYER, «Prédication et État napolitain dans la première moitié du XIV siècle », ibid., p. 127-157.

37. S. Kelly, The New Solomon : Robert of Naples (1309-1343) and FourteenthCentury Kingship, Leyde/Boston, 2003.

38. G.L. BORGHESE, Carlo I d'Angiò e il Mediterraneo.Politica, diplomazia e commercio internazionale prima dei Vespri, Rome, 2008 (Collection de l'École française de Rome, 411). 
à côté d'Andreas Kiesewetter dans le courant dépendant de la reconstruction archivistique. J'ai en effet tenté, dans une étude de fond sur la politique et le commerce extérieur du royaume angevin, de reconsidérer systématiquement le témoignage des sources littéraires, jusqu'alors uniquement utilisées dans l'historiographie courante sur le sujet, à l'aide des données administratives contenues dans les registres et qui jusqu'à présent étaient restées largement inexploitées.

Quid, enfin, des évolutions actuelles de la reconstruction qui conditionneront la pratique future des historiens ? Nous sommes apparemment entrés ces dernières années, après la phase des répertoires et celle des transcriptions, dans une troisième phase, immatérielle, de la reconstruction archivistique des registres angevins. Je fais référence au projet de l'Université Federico II de Naples de construire un système informatique pour la gestion et l'analyse de notre documentation. Roberto Delle Donne a soutenu la nécessité de cette entreprise dès $2001^{39}$, en soulignant que les quarante-cinq volumes des registres jusqu'alors publiés représentaient une entreprise méritoire, mais d'utilisation difficile dans leur version imprimée, tant à cause de la nature hétérogène de leur documentation que de leur incomplétude, et surtout de la fiabilité relative de leurs index. Il a en outre rappelé le caractère nécessairement incomplet de cette reconstruction pour le passé comme pour l'avenir : plusieurs éditions de sources n'auraient pas été comprises, pour divers motifs, dans le dépouillement général (Eduard Sthamer, Romualdo Trifone, Francesco Scandone, Gennaro Maria Monti, Bianca Mazzoleni, Nicola Nicolini et Andreas Kiesewetter lui-même ${ }^{40}$ ) : la publication en 2005, par Serena Morelli, du fonds d'archives de l'historien Léon Cadier, appartenant aujourd'hui à la Bibliothèque nationale de France $^{41}$, rend accessibles, entre autre, ses transcriptions de documents angevins pour la période 1285-1293, qui ne sont pas incluses dans le volume XLV des registres reconstruits, confirmant de la sorte les limites de l'œuvre de reconstruction soulignées par Roberto Delle Donne.

Il subsiste, d'autre part, une documentation originale encore inédite dans certaines archives italiennes ou européennes qui a échappé aux recherches de l'Ufficio della Ricostruzione : je pense en particulier à la documentation produite par Robert II d'Artois, régent du royaume de Sicile de 1285 à 1289, et conservée aux Archives départementales du Pas-deCalais. On peut encore penser à la documentation inédite connue, mais qui

39. Sur le site officiel de l'Université Federico II de Naples, http://www.storia.unina.it/ angio.

40. Mais Stefano Palmieri se montre, à ce propos, d'avis contraire : ID., «I registri...», p. 404, n. 58 .

41. S. MoRelli éd., Le carte di Léon Cadier alla Bibliothèque nationale de France. Contributo alla ricostruzione della Cancelleria angioina, Rome, 2005 (Sources et documents d'histoire du Moyen Âge, 9). 
pose des problèmes méthodologiques particuliers concernant l'inclusion dans les registres reconstruits. C'est notamment le cas des originaux des mandats angevins conservés par les destinataires. Mais, comme le souligne Stefano Palmieri ${ }^{42}$, nous n'avons aucune garantie qu'une transcription des originaux que nous possédons aujourd'hui ait existé au Moyen Âge dans les registres.

La documentation restée en marge de la reconstruction justifie donc le projet universitaire napolitain de créer, par-delà les registres édités, une sorte d'archive digitalisée de tout le matériel récupéré, dans les registres et en dehors d'eux, avec des modalités d'interrogation qui permettent une vision unitaire évitant néanmoins le risque de créer des unités arbitraires, comme il est tant de fois arrivé dans le passé. Destin de nombreux travaux universitaires : le projet en question était présenté comme très avancé par son comité de rédaction en 2004. Il semble encore loin, courant 2014, d'être parfaitement opératoire ${ }^{43}$. Ce fait semble d'autant plus regrettable que le tarissement des financements publics a conduit depuis une quinzaine d'années à un ralentissement progressif de la publication des registres. Mais il ne s'agit là que d'un nouvel avatar d'une histoire tourmentée.

Gian Luca Borghese - Università di Torino - CRISM

Les registres de la chancellerie angevine de Naples : un exemple de destruction et reconstitution de sources archivistiques à travers les siècles

La destruction en 1943 des registres des Archives angevines de Naples est souvent considérée comme un exemple spectaculaire d'appauvrissement irrémédiable des sources d'un grand secteur de l'histoire médiévale. À un " avant " de l'abondance s'oppose emblématiquement un " après " de l'absence, changeant radicalement les conditions de la recherche historique. Une reconstitution détaillée de l'histoire de ces sources dans la longue durée permet montre une réalité plus nuancée. À un processus de destructurationrecomposition progressive, à l'époque médiévale et moderne, succède une première exploitation scientifique polycentrique, interrompue par la catastrophe de 1943. Les tentatives de récupération de cet héritage textuel de 1945 à nos jours à partir de matériaux divers (transcriptions, autres fonds d'archives) sont également caractérisés par une multiplicité de stratégies. Cette histoire textuelle n'est donc pas seulement celle d'une destruction : elle peut apprendre beaucoup sur les conditions paradoxales de la recherche historique.

Chancellerie de Naples - diplomatique - histoire textuelle - registres - Italie angevine

42. S. PALMIERI, «I registri...», p. 402, n. 57.

43. La consultation du site cité $n$. 39 et des différents portails d'accès aux bases de données élaborées par R. Delle Donne semblait se réduire courant 2014 à la page d'introduction, sans que le chercheur extérieur à l'Université de Naples puisse savoir si l'absence de possibilité d'accéder aux bases proprement dites, pourtant présentée comme achevées, résultait d'un réaménagement en cours, de problèmes informatiques, ou d'autres raisons. 


\section{The Registers of the Angevin Archive of Naples : an Example of Destruction} and Reconstruction of Archive's Source through the Centuries

The destruction in 1943 of the Registers of the Angevin Archive of Naples is often considered as a spectacular case of irremediable impoverishment affecting the sources of a large sector of Medieval History. An ancient time of archival abundance is thus emblematically opposed to a new period of absence, with a dramatic change for the conditions of scholarly research. A detailed investigation of the "longue durée" history of these sources allows us to adopt a more nuanced approach. Already during the Middle Ages and the early modern history, the Angevin Registers were the object of complex processes of de-structuration/restructuration. They were followed by a first wave of polycentric scientific exploitation that was interrupted by the catastrophe of 1943. The attempts to reconstruct this textual heritage, from 1945 to the present days, with the help of a vast array of sources (early transcripts, other archives, etc...), have been equally characterized by a multiplicity of strategies. This textual history is consequently not only the history of a violent collapse. It can also teach us a lot on the paradoxical conditions that govern research in history.

Angevin Italy - diplomatic - Neapolitan Chancery - registers - textual history 